\title{
Metallo- $\beta$-lactamase-producing Pseudomonas aeruginosa in two hospitals from Southern Brazil
}

\author{
Wirth, Fernanda W. ${ }^{1}$, Picoli, Simone U. ${ }^{1}$, Cantarelli, Vlademir V. ${ }^{1,2}$, Gonçalves, Ana L.S. ${ }^{1}$, Brust, Flávia R. ${ }^{3}$, Santos, Liege M.O. ${ }^{3}$ \\ and Barreto, Michelle $\mathrm{F}^{4}$ \\ ${ }^{1}$ Feevale, Laboratory of Biomedicine, Novo Hamburgo, RS; ${ }^{2}$ Weinmann Laboratory, Porto Alegre, RS; ${ }^{3}$ Exame Laboratory, Novo Hamburgo; \\ RS; ${ }^{4}$ Unilab, Porto Alegre, RS; Brazil
}

\begin{abstract}
This study determined the prevalence of metallo- $\beta$-lactamase (MBL)-producing Pseudomonas aeruginosa in two hospitals located in the Southern part of Brazil and compare the performance of two different phenotypic tests. Thirty-one non-repetitive Pseudomonas aeruginosa isolates from various clinical samples from patients admitted to two hospitals located in Rio Grande do Sul, Brazil (twenty-three from a hospital in Porto Alegre City and eight isolates from a hospital in Vale dos Sinos Region). All strains suggestive of possessing MBLs by phenotypic methods were included in this study. Phenotypic detection of MBLs was carried out simultaneously by using both the MBL Etest ${ }^{\circledR}$ and disk approximation test using 2-mercaptopropionic acid close to a ceftazidime disk. Strains positive were further confirmed using molecular techniques for bla $_{\mathrm{VIM}}$, bla $_{\mathrm{IMP}}$ and $b l a_{\mathrm{SPM}-1}$. The prevalence of MBLs from samples of inpatients from the hospital located in Porto Alegre was $\mathbf{3 0 . 4 \%}$ and that of inpatients from Vale dos Sinos hospital was only 3.1\%. Only MBL type SPM-1 was detected in these samples by molecular analysis and all were detected by the Etest ${ }^{\circledR}$ MBL strips. The prevalence of $P$. aeruginosa that produce MBLs can be markedly different in distinct geographical areas, even among different hospitals in the same area. In our study, the EDTA-based method was the only method able to detect all strains harboring the SPM-1 enzyme.
\end{abstract}

Key-Words: Metallo-beta-lactamase, Pseudomonas aeruginosa, multiresistance.

Pseudomonas aeruginosa is a Gram-negative rod unable to ferment glucose and is widely known as an opportunistic organism, frequently involved in infections of immunosuppressed patients, and also causes outbreaks of hospital-acquired infections [1]. P. aeruginosa is very commonly isolated from nosocomial infections and is the most prevalent bacterial agent isolated from cases of pneumonia in Brazilian hospitals. This organism is clinically important since it possesses several virulence factors and is intrinsically resistant to most antimicrobial and disinfectant agents, a feature that is also responsible for the difficulty in treating infected patients [2].

Carbapenems are generally used as an option to treat serious infections caused by $P$. aeruginosa, since these drugs present a good spectrum of activity and are stable to hydrolysis by most $\beta$-lactamases, including the extended spectrum b-lactamases (ESBL). However, the use of carbapenems has been hampered by the emergence of strains that produce metallo- $\beta$-lactamase (MBL), an enzyme that is able to hydrolyze and inactivate this class of antibiotics. Moreover, the spread of MBL-producing clones, even over distant regions, has been reported [2].

Among $\beta$-lactamases, MBLs are unique in requiring the presence of zinc ion in the active site of the enzyme, and are, thus, inhibited by chelating agents such as EDTA [3].

Since the early 90s, new genes coding for distinct MBLs have been described in clinical important pathogens like Pseudomonas spp., Acinetobacter spp. and even among members of the family Enterobacteriaceae. These genes are

Received on 28 February 2009; revised 12 May 2009.

Address for correspondence: Dr. Fernanda Wirth. Centro Universitário Feevale, Laboratório de Biomedicina, RS 239 n²755, CEP 93525000, Vila Nova, Novo Hamburgo, RS, Brazil. Phone 555135868800.

The Brazilian Journal of Infectious Diseases 2009;13(3):170-172. (C) 2009 by The Brazilian Journal of Infectious Diseases and Contexto Publishing. All rights reserved. usually inserted in mobile elements facilitating the exchange of these resistance genes among several bacterial species [4]. Several MBLs were reported, including: IMP, VIM, SPM, GIM and, more recently, SIM-1, encoded by the $b l a_{\mathrm{SIM}-1}$ gene in isolates of A. baumannii from South Korea [5].

Prompt detection and recognition of the MBLs is important to implement adequate counter-measures to control the spread of the organisms bearing these enzymes, and proper treatment of infections caused by MBL-producing microorganisms. A few phenotypic methods have been published for MBL detection; however, the results have shown that no method alone is able to detect all these enzymes, probably due to the genetic variability of these enzymes [6]. Molecular methods are more sensitive and specific for the detection of MBLs, but they may still be technically demanding and non available in most clinical microbiology laboratories.

We sought to determine the prevalence of MBL in $P$. aeruginosa strains from two South Brazilian hospitals and compare the efficacy of two different phenotypic methods to detect MBLs in this organism.

\section{Material and Methods}

Thirty-one clinical isolates of carbapenem-resistant $P$. aeruginosa were collected from two hospitals in Southern Brazil. Identification of the isolates was confirmed using conventional techniques [7] and disk diffusion susceptibilities were determined according to the CLSI methodology [8]. Only one isolate per patient was included and those isolates from cystic fibrosis patients were excluded from this study.

The following antimicrobial agents were included: aztreonam (ATM), cefepime (CPM), ceftazidime (CAZ) and imipenem (IPM), all from Cecon, São Paulo, SP, Brazil. Strains classified as resistant or intermediate to these drugs were included in this study. The production of MBL was screened for using two different methods, simultaneously: (i) using the 
approximation test with CAZ disk close to a 2mercaptopropionic acid (2-MPA, Sigma-Aldrich, Buchs, United States) and $3 \mu \mathrm{L}$ of the original reagent was used to impregnate a blank disk, exactly as described by Arakawa et al. (2000) [9]. The 2-MPA-containig disk was placed $25 \mathrm{~mm}$ apart (center to center) from a ceftazidime disk on a Mueller-Hinton agar plate. An inhibition zone observed close to these disks were considered positive for the presence of MBL.

The second method consisted of an Etest ${ }^{\circledR}$ MBL-detection strip (AB Biodisk, Dalvägen, Solna, Sweden) placed onto the same agar plate, which was considered positive for MBL whenever the minimal inhibitory concentration (MIC) of imipenem plus EDTA decreased at least three dilutions as compared to the imipenem MIC alone [10]. A strain of $P$. aeruginosa known to produce SPM-1 metallo-beta-lactamase was used as a positive control in all cases. This strain has previously been characterized in Laboratório Weinmann (Porto Alegre, Brazil) by PCR with primers specific for $b l a_{\mathrm{SPM}}$, as described by Gales et al. (2003) [2], and further confirmed by direct DNA sequencing of the generated PCR products. A MBL negative strain (ATCC 27853; distributed by Oxoid, Basingstoke, Hampshire, England) was also used as a negative control.

All strains suggestive of possessing MBLs by either of the phenotypic tests were further confirmed using molecular techniques. SPM-1 was detected using the polymerase chain reaction (PCR) and specific primers as described above. Two other clinical strains of $P$. aeruginosa positive for MBL and confirmed to harbor bla $a_{\mathrm{VIM}}$ and bla ${ }_{\mathrm{IMP}}$ by specific PCR, as described previously by Pitout et al. [11], and further checked by DNA sequencing. All PCR tests were conducted in appropriated rooms to prevent cross-contamination and proper positive and negative controls were used all the time.

\section{Results}

Eight $P$. aeruginosa strains isolated from the Vale dos Sinos hospital fulfilled the criteria for inclusion in this study: resistance to CAZ, CPM and/or IPM. Among these 3 (37.5\%) were considered resistant and 1 (12.5\%) intermediate to ceftazidime, 4 (50\%) were resistant to cefepime, 2 (25\%) resistant to aztreonam and only 1 (12.5\%) showed resistance to imipenem.

The frequency of MBL in this institution was considered low (3.1\%), and the single MBL-positive strain was detected by the Etest-MBL $\AA$ alone. None of the isolates were from intensive care unit (ICU) patients. The isolates were from diverse clinical samples, such as urine (50\%), sputum (25\%), tracheal aspirates (12.5\%) and skin ulcers (12.5\%). The MBLpositive $P$. aeruginosa strain was from a skin ulcer and presented classical phenotypical features compatible with the presence of MBL, i. e., resistance to CAZ, CPM and IPM and susceptibility to ATM.

Among the 23 strains isolated in Porto Alegre, 7 (30.4\%) showed phenotypic evidences of MBL production and all were detected only with the E-test method (Table 1). Thirteen strains were isolated from ICU patients (56.5\%), and among these, 5 were positive for MBL production; however, this finding was not considered statistically significant by the Qui-square test.

The strains of $P$. aeruginosa isolated in Porto Alegre were from clinical samples such as tracheal aspirates (69.6\%), sputum (17.4\%), urine (8.7\%) and one was from an unknown source $(4.3 \%)$.

Interestingly, none of the $7 \mathrm{MBL}$-producing strains could be detected by the CAZ/2-MPA disks, as proposed by Arakawa et al. (2000) [9]. However, all strains were considered positive for MBL production by the E-test strip.

According to Marra et al. (2006) [12] and Magalhães et al. (2005) [13], the SPM-1 enzyme, which is highly prevalent in Brazil, could be equally detected by the 2-MPA method and the EDTA-based method. Zavascki et al. (2005) [14] reported that $77.1 \%$ of their $P$. aeruginosa isolates, resistant to IPM, could be detected by 2-MPA and $87.5 \%$ of the strains carried the bla $a_{\mathrm{SPM}-1}$ gene. Similarly, another study by Zavascki et al. (2006) [15] reported the detection of $28.9 \%$ of MBL-positive $P$. aeruginosa, containing the bla $a_{\mathrm{SPM}-1}$ gene, when the 2-MPA method was used to screen for the presence of this enzyme. In our study, 19.3\% (6 strains) were positive for the presence of SPM-1, as confirmed by PCR analysis, but none could be detected using the phenotypic test proposed by Arakawa et al. (2000) [9]. Interestingly, in the remaining 2 strains, which were clearly MBL positive by phenotypic test (Etest), no other betalactamase gene could be found by PCR as specific primers (bla $a_{\mathrm{SPM}-1,}$ bla $a_{\mathrm{IMP}}$ or $b l a_{\mathrm{VIM}}$ ), suggesting that maybe another kind of MBL gene(s) may be involved in these strains. It is known that EDTA may increase bacterial cell-wall permeability and that zinc (chelated by EDTA) accelerates imipenem decomposition and decreases OprD expression of $P$. aeruginosa [16]. Another alternative would be the presence of OXA-10 and/or OXA-14 stabilized in the dimeric form by metal ions $\left(\mathrm{Zn}^{++2}\right)$. Dimeric forms are more active than the monomeric form, increasing its enzymatic activity turning the enzyme more efficient to inactivate carbapenems and other beta-lactam antibiotics. However, in the presence of a chelator agent, such as EDTA, the OXA class of enzymes is converted to their monovalent forms, which are less efficient in their activity against carbapenems. For these reasons, it is advisable to be cautious when interpreting the results of tests that use EDTA, as the E-Test MBL [17].

Detailed molecular knowledge of the MBLs involved may be necessary to better understand the reasons for success or failure of the phenotypic methods used for MBL screening.

Considering the susceptibility profile of our isolates, all MBL-positive samples were resistant to IPM, CAZ and CPM, including three of these samples also resistant to ATM. The possible explanation for this event is the concomitant presence of another extended-spectrum beta-lactamase (Such as the Ambler's class A). Similar results were found in a work by Marra and coworkers (2006) [12], where, among four $P$. aeruginosa isolates positive for SPM-1, only two were resistant to ATM, while all strains were resistant to CPM and IPM. In this study, the authors also found isolates harboring the IMP-16, which were equally resistant to ATM. 
Table 1. Clinical and microbiological characteristics of imipenem-resistant clinical isolates of Pseudomonas aeruginosa.

\begin{tabular}{|c|c|c|c|c|c|c|}
\hline Strain & Hospital & Sample & $\begin{array}{c}\text { Diffusion disk }^{\mathrm{a}} \\
\text { ATM/CAZ/CFP/IMP }\end{array}$ & DDT $^{b}$ & $\begin{array}{c}\text { E-Test }^{\mathrm{c}} \\
\text { IP/IP+EDTA } \geq 8\end{array}$ & $\begin{array}{c}\text { PCR } \\
\text { bla gene }\end{array}$ \\
\hline 1 & VS & skin ulcer & $S / R / R / R$ & - & + & SPM \\
\hline 2 & PA & tracheal aspirate & $\mathrm{R} / \mathrm{R} / \mathrm{R} / \mathrm{R}$ & - & + & SPM \\
\hline 3 & PA & tracheal aspirate & $\mathrm{R} / \mathrm{R} / \mathrm{R} / \mathrm{R}$ & - & + & SPM \\
\hline 4 & PA & sputum & $S / R / R / R$ & - & “Ghost zone” & SPM \\
\hline 5 & PA & tracheal aspirate & $\mathrm{R} / \mathrm{R} / \mathrm{R} / \mathrm{R}$ & - & + & SPM \\
\hline 6 & PA & tracheal aspirate & $S / R / R / R$ & - & + & SPM \\
\hline Control & - & - & $S / R / R / R$ & + & + & SPM \\
\hline
\end{tabular}

${ }^{a} A T M$ : aztreonam; CAZ: ceftazidime; CFP: cefepime; IMP: imipenem. S: susceptible, R: resistant. ${ }^{b}$ Double-disk test (DDT) was performed using disks containing the 2-mercaptopropionic acid and ceftazidime as inhibitor and substrate, respectively. $(-)$ negative; $(+)$ positive. ${ }^{\text {TThe }}$ MBL presence is considered when MIC IP/IP+EDTA $=8$. Ghost zone: suggestive of MBL production.

VS: Vale dos Sinos; PA: Porto Alegre.

In the present study, among the strains negative for the presence of MBLs, two were resistant only to IMP. The susceptibility to the others $\beta$-lactamic agents, such as CAZ, and resistance to IMP could be explained by a mechanism involving porin loss. According to Troillet et al. (1997) [18], this discrepancy can be explained by the fact that carbapenems use a specific porin to enter the bacterial cell (porin OprD), while other $\beta$-lactamic agents use other kinds of porins. In this way, the loss of OprD alone would result in an increased MIC for IMP only. In this case, it would be possible to use the results of the susceptibility test to guide the therapeutic approach used to treat the infected patient.

\section{Conclusion}

In conclusion, we demonstrated here that some strains of $P$. aeruginosa producing the SPM- 1 type of MBL are not detected by phenotypic tests based on the disk-approximation method (e.g. CAZ plus 2-MPA), suggesting that the use of EDTA-based tests, or a combination of tests, would be preferred to increase the sensitivity of these methods to detect the presence of MBL among clinical isolates of $P$. aeruginosa.

\section{Acknowledgements}

Our thanks to Alessandra Navarini from Probac do Brasil for kindly supplying the MBL-Etest ${ }^{\circledR}$ strips, and to Jaqueline Bohrer Schuh for the statistical analysis.

\section{References}

1. Sader H.S., Gales A.C., Pfaller M.A. et al. Pathogen frequency and resistance patterns in Brazilian hospitals: summary of results from three years of the SENTRY Antimicrobial Surveillance Program. Braz J Infect Dis 2001;5:200-14.

2. Gales A.C., Menezes L.C., Silbert S., Sader H.S. Dissemination in distinct Brazilian regions of an epidemic carbapenem-resistant Pseudomonas aeruginosa producing SPM metallo-betalactamase. J Antimicrob Chemother 2003;52:699-702.

3. Bush K., Jacoby G.A., Medeiros A.A. A functional classification scheme for beta-lactamases and its correlation with molecular structure. J Antimicrob Chemother 1995;39:1211-33.

4. Luzzaro F., Endimiani A., Docquier J.D. et al. Prevalence and characterization of metallo-beta-lactamases in clinical isolates of Pseudomonas aeruginosa. Diagn Microbiol Infect Dis 2004;48:131-5.
5. Mendes R.E., Castanheira M., Pignatari A.C.C., Gales A.C. Metalo$\beta$-lactamases. J Bras Patol Med Lab 2006;42:103-13.

6. Martins A.F. Caracterização de metalo- $\beta$-lactamases produzidas por amostras de Pseudomonas aeruginosa isoladas em dois hospitais de Porto Alegre. Porto Alegre, 2005, 58p. (Dissertação de Mestrado. Programa de Pós-Graduação em Ciências Farmacêuticas. UFRGS).

7. Kiska D.L., Gilligan P.H. Pseudomonas. In: Murray P.R., Baron E.J., Pfaller M.A., Tenover F.C., Yolken R.H. (edts). Manual of Clinical Microbiology. 7th ed. Washington: American Society for Microbiology, 1999.

8. Clinical and Laboratory Standards Institute (CLSI). Performance standards for antimicrobial susceptibility testing. Sixteenth international supplement, M100 - S16. Wayne, 2006.

9. Arakawa Y., Schibata N., Shibayama K. et al. Convenient Test for Screening Metallo- $\beta$-Lactamase-Producing Gram-Negative Bacteria by Using Thiol Compounds. J Clin Microbiol 2000;38:40-3.

10. Walsh T.R., Bolmstrom A, Qwarnstrom A, Gales A. Evaluation of a new Etest for detecting metallo- $\beta$-lactamases in routine clinical testing. J Clin Microbiol 2002;40:2755-9.

11. Pitout J.D.D., Gregson D.B., Poirel L., McClure J.A., Church D.L. Detection of Pseudomonas aeruginosa Producing Metallo-âLactamases in a Large Centralized Laboratory. J Clin Microbiol 2005;43:3129-35.

12. Marra A.R., Pereira C.A., Gales A.C. et al. Bloodstream infections with metallo-b-lactamase-producing Pseudomonas aeruginosa: epidemiology, microbiology and clinical outcomes. Antimicrob Agents Chemothe. 2006;50:388-90.

13. Magalhães V., Lins A.K., Magalhães M. Metallo- $\beta$-lactamase producing Pseudomonas aeruginosa strains isolated in hospitals in Recife, PE, Brasil. Braz J Microbiol 2005;36:123-125.

14. Zavascki A. P., Gasparetto P.B., Martins A.F., et al. Outbreak of carbapenem-resistant Pseudomonas aeruginosa producing SPM1 metallo-beta-lactamase in a teaching hospital in southern Brazil. J. Antimicrob. Chemother. 2005;56:1148-51.

15. Zavascki A.P., Barth A.L., Gonçalves A.L.S. et al. The influence of metallo-ß-lactamase production on mortality in nosocomial Pseudomonas aeruginosa infections. J Antimicrob Chemother 2006;58:387-92.

16. Conejo M.C., Garcia I., Martinez-Martinez L. et al. Zinc eluted from siliconized latex urinary catheters decreases OprD expression, causing carbapenem resistance in Pseudomonas aeruginosa. Antimicrob Agents Chemother 2003;47:2313-5.

17. Danel F., Paetzel M., Strynadka N.C. Effect of divalent metal cations on the dimerization of OXA-10 and -14 class D $\beta$-lactamases from Pseudomonas aeruginosa. Biochemistry 2001;40:9412-20.

18. Troillet N., Samore M.H., Carmeli Y. Imipenem-resistant Pseudomonas aeruginosa: risk factors and antibiotic susceptibility patterns. Clin Infect Dis 1997;25:1094-8. 